\title{
Coupling, resonance transmission, and tunneling of surface-plasmon polaritons through metallic gratings of finite length
}

\author{
Jose A. Sánchez-Gil \\ Instituto de Estructura de la Materia, Consejo Superior de Investigaciones Científicas, Serrano, 121, 28006 Madrid, Spain
}

(Received 5 October 1995)

\begin{abstract}
The Green's-theorem integral equation formulation is employed to study numerically the coupling into surface plasmon polaritons by illuminating a finite metal grating with a Gaussian beam from the vacuum half-space above the metal surface. A flat surface impedance boundary condition is used to simplify the scattering integral equations. The grating coupler period is chosen so that the first diffracted order excites a surface plasmon polariton and the zeroth diffracted order is the only radiating order. In particular, the surface magnetic field and the angular distribution of scattered intensity are calculated. These functions provide in turn the total intensity of the radiation scattered into the vacuum and the total power flow carried by the surface plasmon polariton, from which the energy balance is monitored. In this way, the zeroth order and coupling efficiencies are studied as functions of the angle of incidence $\theta_{0}$ and the grating coupler height $s_{c}$, with the aim of analyzing the influence of the length of the illuminated coupler. Our results show that, when the illuminated coupler length is decreased, the photon-surface plasmon polariton coupling resonance broadens as a function of both $\theta_{0}$ and $s_{c}$, and that larger values of $s_{c}$ are required to optimize this coupling resonance. In addition, the coupling geometry is exploited to obtain the reflection and transmission coefficients, and the intensity of the scattered volume waves, of a surface plasmon polariton thus excited that impinges on another finite metal grating, called a grating scatterer. Two frequencies of the incoming surface plasmon polariton are considered that lie very close to the lower band edge of the gap in the surface plasmon polariton dispersion relation for the infinite grating scatterer. If the frequency is in the band, a strongly oscillating, resonant behavior of the transmission coefficient as a function of the grating length is obtained. For a frequency in the gap, transmission is negligible unless the grating is short enough that the surface plasmon polariton can tunnel through it.
\end{abstract}

\section{INTRODUCTION}

The subject of diffraction of electromagnetic waves by gratings is a classical one. The existence of anomalies in the spectral and angular dependence of the various diffracted orders has attracted a great deal of attention ever since the observation by Wood ${ }^{1}$ in 1902 of such anomalies for $p$ polarization (magnetic field vector parallel to the grooves, TM) in experiments on shallow metal gratings. Lord Rayleigh ${ }^{2}$ presented in 1907 the first theoretical treatment of these anomalies, which prompted a number of subsequent studies in the following decades based on the so-called Rayleigh method. Among them, the work by $\mathrm{Fano}^{3}$ represented an important step forward, for it first indicated the existence of two types of anomalies. But it was not until the work by Hessel and Oliner, ${ }^{4}$ by means of an idealized surface reactance model of a planar periodic structure, that those $p$-type anomalies were correctly understood. They distinguished Rayleigh anomalies, ${ }^{2}$ occurring when one of the diffracted orders emerges from the grating at a grazing angle, from those resonance anomalies appearing when an evanescent order excites a surface plasmon polariton (SPP) supported by the corrugated metal interface.

In recent years, a great variety of rigorous vector theories of gratings have been developed for either perfectly conducting or finite conductivity materials (cf. Refs. 5 and 6), which can be grouped into differential and integral methods. Several works have been devoted to the anomalies originating in the grating-induced coupling of light into a SPP (Refs.
7-12), with special emphasis on the occurrence of both total absorption of light and large surface electromagnetic field enhancements, and their implications in surface-enhanced Raman spectroscopy and other nonlinear phenomena. ${ }^{8,9,13}$ In particular, the integral equation methods have been proven to be more appropriate for analyzing gratings with continuous profiles, and capable of giving very accurate numerical results even for deep and inhomogeneous gratings. ${ }^{14-17} \mathrm{Up}$ to the present, most of the existing theoretical formulations have exploited the pseudoperiodicity of the electromagnetic field inherently associated with the infinite extent of the grating. Nonetheless, in many instances, the conditions of grating illumination and geometry make it necessary to develop a diffraction theory that deals with finite gratings (cf., for example, Ref. 18 and references therein). In addition, the possibility of combining different gratings of finite length into a single device, which might be of great interest in many practical applications in diffractive and integrated optics, also suggests the need of such theories.

For that purpose, we have applied in this paper the rigorous Green's-theorem integral equation formulation, used in the scattering of electromagnetic waves from rough metal or dielectric surfaces, ${ }^{19-23}$ to determine the field scattered by a one-dimensional metal surface consisting of two sections of sinusoidal gratings with different periods separated by a planar section (a similar configuration has been treated by Saillard, ${ }^{23}$ but the emphasis there was put on the qualitative aspects of SPP localization in random media). A Gaussian beam impinges on one of the gratings (see Fig. 1), whose period is such that a forward propagating SPP is excited 


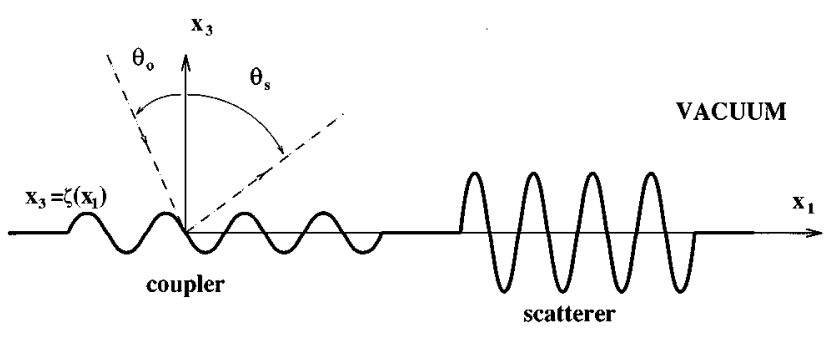

METAL

FIG. 1. Illustration of the scattering geometry.

whereas only the zeroth order is diffracted into the vacuum above the metal surface. The SPP thus excited then impinges on a second grating; as a result, it is partially reflected, partially transmitted, and partially converted into volume waves in the vacuum region. The period of the second grating is chosen so that the frequency of the incoming SPP lies in the vicinity of the lower band edge of the gap in the SPP dispersion relation. In what follows, we will refer to the two gratings as coupler and scatterer, respectively. The numerical treatment of the formulation allows us to obtain the scattered electromagnetic field throughout the entire space. In particular, we focus on the far-field radiated intensity and the surface field. In addition, the energy carried by the SPP is also calculated, so that the energy balance is accurately determined. In this manner, we study the finite-scale effects in the coupling and scattering of SPP. First, as regards photon-SPP coupling, the dependence of the diffracted and coupling efficiencies on the angle of incidence and grating depth is analyzed for both an ideal lossless metal and for silver. Second, we are concerned with the behavior of the SPP reflection and transmission coefficients, properly normalized for a lossless metal, as a function of the number of grating scatterer periods for frequencies both in the band and in the gap of the SPP band structure of the infinite grating.

It should be remarked that the motivation for this work, in addition to its above-mentioned relevance in the diffractive optics of metallic gratings, stems from the interest in the propagation properties of SPP, ${ }^{24,25}$ especially with regard to their scattering and localization on corrugated metal interfaces (cf. Refs. 23 and 26-28). Furthermore, much effort has been concentrated on the experimental and theoretical investigation of these problems in connection with the recent development of near-field optical microscopy. ${ }^{29-33}$ On the other hand, the configurations studied here could be extended to address equivalent configurations for guided-wave polaritons in dielectric gratings, which are of significant importance in related applications such as couplers, filters, and distributedfeedback lasers.

Finally, we would like to point out that a local impedance boundary condition on a planar surface has been used to simplify the set of integral equations upon which the formulation is based. In this manner, the electromagnetic field inside the metal is eliminated from the calculation, thus leading to a considerable reduction of computation time in the numerical simulations. Recently, Maradudin ${ }^{34}$ has shown the connection of such a surface impedance with the real surface corrugation as an expansion in powers of the surface profile function, which is valid for the frequencies and shallow grat- ings (groove depth to period ratios lower than 0.5) we are interested in. However, if deeper gratings were to be studied, one could either use local ${ }^{35,36}$ and nonlocal ${ }^{37}$ impedance boundary conditions at a curved surface, which are not restricted to smooth corrugations, or leave the rigorous scattering integral equations unaltered (so that the formulation is intrinsically exact).

The paper is organized as follows. In Sec. II, the configuration studied in this work is defined, and the integral equation formulation is described. In Sec. III, we present the numerical results for the coupling of a Gaussian beam into SPP through a finite metal grating. The calculations for the scattering of a SPP by a finite section of a grating under conditions close to Bragg reflection are shown in Sec. IV. Finally, Sec. V contains the conclusions drawn from the results obtained.

\section{FORMULATION OF THE PROBLEM}

\section{A. Scattering equations}

Let us consider a one-dimensional rough interface $x_{3}=\zeta\left(x_{1}\right)$ separating vacuum $\left[x_{3}>\zeta\left(x_{1}\right)\right]$ from a metal $\left[x_{3} \leqslant \zeta\left(x_{1}\right)\right]$ characterized by an isotropic, frequencydependent dielectric function $\varepsilon(\omega)$. A monochromatic, $p$-polarized electromagnetic (EM) wave whose plane of incidence is the $x_{1} x_{3}$ plane impinges on the surface. Under these conditions, the problem is fully described by means of the transverse component of the magnetic field, $\mathrm{H}_{2}\left(x_{1}, x_{3}\right)$, whose amplitude obeys the two-dimensional Helmholtz equation:

$$
\begin{gathered}
\left(\frac{\partial^{2}}{\partial x_{1}^{2}}+\frac{\partial^{2}}{\partial x_{3}^{2}}+\frac{\omega^{2}}{c^{2}}\right) H_{2}^{>}\left(x_{1}, x_{3}\right)=0, \quad x_{3}>\zeta\left(x_{1}\right) \\
\left(\frac{\partial^{2}}{\partial x_{1}^{2}}+\frac{\partial^{2}}{\partial x_{3}^{2}}+\varepsilon(\omega) \frac{\omega^{2}}{c^{2}}\right) H_{2}^{<}\left(x_{1}, x_{3}\right)=0, \quad x_{3} \leqslant \zeta\left(x_{1}\right) .
\end{gathered}
$$

The superscripts $>$ and $<$ indicate inside vacuum and metal, respectively. It has been shown in Refs. 19-22 that this problem can be cast into the form of four integral equations through the use of Green's theorem. These integral equations relate $H_{2}^{>}\left(x_{1}, x_{3}\right)$ and $H_{2}^{<}\left(x_{1}, x_{3}\right)$ to the magnetic field and its normal derivative evaluated at the interface, which in turn play the role of source functions. With the aid of the continuity conditions across the interface, and on using the integral equations as extended boundary conditions, these source functions can be obtained by numerically solving a set of two coupled integral equations (see Refs. 19 and 21). However, instead of proceeding in that manner, we further simplify the formulation by mapping the continuity conditions at the corrugated surface $x_{3}=\zeta\left(x_{1}\right)$ onto a local impedance boundary condition on the planar surface $x_{3}=0$ as follows:

$$
\begin{aligned}
& \left.\frac{\partial}{\partial x_{3}} H_{2}^{>}\left(x_{1}, x_{3}\right)\right|_{x_{3}=0} \\
& \quad=-\left.\frac{\omega}{c}[-\varepsilon(\omega)]^{-1 / 2}\left[1+s\left(x_{1}\right)\right] H_{2}^{>}\left(x_{1}, x_{3}\right)\right|_{x_{3}=0},
\end{aligned}
$$


where $s\left(x_{1}\right)$ is the surface impedance. It has been demonstrated by Maradudin ${ }^{34}$ that the right-hand side of Eq. (2) corresponds to the zeroth- and first-order terms of an expansion in powers of the surface profile function $\zeta\left(x_{1}\right)$, so that

$$
s\left(x_{1}\right)=-\frac{1-\varepsilon(\omega)}{\varepsilon(\omega)} \frac{1}{d(\omega)}\left[1-d^{2}(\omega) D^{2}\right]^{1 / 2} \zeta\left(x_{1}\right)+O\left(\zeta^{2}\right),
$$

where $D \equiv d / d x_{1}$, and $d(\omega) \equiv c[-\varepsilon(\omega)]^{-1 / 2} / \omega$ is the skin depth. We will not discuss here the constraints on this connection between the real corrugation $\zeta\left(x_{1}\right)$ and the surface impedance $s\left(x_{1}\right)$ (cf. Ref. 34); for our purpose, it suffices to know that boundary condition (2) describes the metallic character of the interface for moderate corrugations and a wide range of frequencies for which the optical skin depth $d(\omega)$ is small compared to the wavelength. We will refer hereafter to the surface impedance rather than to the surface profile function.

With the help of Eq. (2) we restrict the analysis to the magnetic field in the vacuum half-space, which can be shown to satisfy the following integral equation:

$$
\begin{aligned}
H_{2}^{>}(\mathbf{r})= & H_{2}^{(i)}(\mathbf{r})+\frac{1}{4 \pi} \int_{-\infty}^{\infty} d x_{1}^{\prime} H\left(x_{1}^{\prime}\right)\left[\frac{\partial G_{0}\left(\mathbf{r}, \mathbf{r}^{\prime}\right)}{\partial x_{3}^{\prime}}\right. \\
& \left.+\frac{\omega}{c}[-\varepsilon(\omega)]^{-1 / 2}\left[1+s\left(x_{1}^{\prime}\right)\right] G_{0}\left(\mathbf{r}, \mathbf{r}^{\prime}\right)\right],
\end{aligned}
$$

where $H_{2}^{(i)}$ is the amplitude of the incident wave, $\mathbf{r} \equiv\left(x_{1}, x_{3}\right)$ and $\mathbf{r}^{\prime} \equiv\left(x_{1}^{\prime}, x_{3}^{\prime}=0\right) . G_{0}\left(\mathbf{r}, \mathbf{r}^{\prime}\right)$ is the twodimensional free-space Green's function,

$$
G_{0}\left(\mathbf{r}, \mathbf{r}^{\prime}\right)=\pi i H_{0}^{(1)}\left(\frac{\omega}{c}\left|\mathbf{r}-\mathbf{r}^{\prime}\right|\right),
$$

where $H_{0}^{(1)}$ is the zeroth-order Hankel function of the first kind. In Eq. (4) we have defined the source function $H\left(x_{1}\right)$ as the surface magnetic field:

$$
H\left(x_{1}\right)=H_{2}^{>}\left(x_{1}, x_{3}=0\right) .
$$

In order to determine $H\left(x_{1}\right)$, we make $x_{3} \rightarrow 0$ in Eq. (4) so that

$$
\begin{aligned}
H\left(x_{1}\right)= & H_{2}^{(i)}\left(x_{1}, x_{3}=0\right)+\frac{1}{4 \pi} \int_{-\infty}^{\infty} d x_{1}^{\prime} H\left(x_{1}^{\prime}\right)\left[\frac{\partial G_{0}}{\partial x_{3}^{\prime}}\right. \\
& \left.+\frac{\omega}{c}[-\varepsilon(\omega)]^{-1 / 2}\left[1+s\left(x_{1}\right)\right] G_{0}\right]_{x_{3}=0}
\end{aligned}
$$

This Fredholm integral equation of the second kind is then converted into a matrix equation by means of a quadrature scheme that truncates the surface at a length $L$ divided into $N$ points, following a procedure analogous to that of Refs. 19 and 21. Once Eq. (4) is numerically solved, the magnetic field $H_{2}^{>}\left(x_{1}, x_{3}\right)$ is obtained by introducing $H\left(x_{1}\right)$ into Eq. (4). By expanding the Green's function in Eq. (4), the scattered field can be written in the form

$$
H_{2}^{>}\left(x_{1}, x_{3}\right)=\int_{-\infty}^{\infty} \frac{d q}{2 \pi} R_{p}(q, \omega) \exp \left[i q x_{1}+i \alpha_{0}(q, \omega) x_{3}\right]
$$

where

$$
\alpha_{0}(q, \omega)= \begin{cases}\left(\frac{\omega^{2}}{c^{2}}-q^{2}\right)^{1 / 2}, & |q| \leqslant \frac{\omega}{c} \\ i\left(q^{2}-\frac{\omega^{2}}{c^{2}}\right)^{1 / 2}, & |q|>\frac{\omega}{c}\end{cases}
$$

and the scattering amplitude is given in terms of $H\left(x_{1}\right)$ by

$$
\begin{aligned}
R_{p}(q, \omega)= & \frac{1}{2 \alpha_{0}(q, \omega)} \int_{-\infty}^{\infty} d x_{1}^{\prime} e^{-i q x_{1}}\left[\alpha_{0}(q, \omega)\right. \\
& \left.+i \frac{\omega}{c}[-\varepsilon(\omega)]^{-1 / 2}\left[1+s\left(x_{1}\right)\right]\right] H\left(x_{1}\right) .
\end{aligned}
$$

Equations (5) and (8) constitute the basis of our scattering problem.

\section{B. Scattering geometry}

Our aim in this work is to study the coupling of an incident wave into a SPP through a finite grating coupler, and then the scattering of the SPP by a second finite grating of distinct period. To that end, we assume the surface impedance to be (see Fig. 1)

$$
\begin{aligned}
s\left(x_{1}\right)= & s_{c} \cos \left(\frac{2 \pi\left(x_{1}-x_{0}\right)}{d_{c}}\right) \operatorname{thf}\left(x_{1}-x_{0}, L_{c}\right) \\
& +s_{s} \cos \left(\frac{2 \pi x_{1}}{d_{s}}\right) \operatorname{thf}\left(x_{1}, L_{s}\right)
\end{aligned}
$$

with $x_{0}=-L_{s} / 2-L_{f}-L_{c} / 2$. It consists of a sinusoidal grating coupler, centered at $x_{1}=x_{0}$, of period $d_{c}$, height $s_{c}$, and length $L_{c}$, separated from another sinusoidal grating, centered at $x_{1}=0$, of period $d_{s}$, height $s_{s}$, and length $L_{s}$, by a flat segment of length $L_{f}$. Two additional flat segments of length $L_{f}$ are included at the leftmost and rightmost ends of the surface impedance. The function $\operatorname{thf}\left(x_{1}, W\right)$ is used to smooth the boundaries of the finite gratings:

$$
\operatorname{thf}\left(x_{1}, L\right) \equiv \frac{2 \cosh ^{2}(\beta L / 4)}{\cosh (\beta L / 2)+\cosh \left(\beta x_{1}\right)}
$$

To provide sharp enough boundaries, $\beta=100 / L$ in all of our calculations.

Let us consider a Gaussian beam of half-width $W$ incident at the center of the grating coupler $x_{0}$ making an angle $\theta_{0}$ measured counterclockwise with respect to the $x_{3}$ axis. The half-width of its intercept with the plane $x_{3}=0$ is $g=W / \cos \theta_{0}=L_{c} / 4$, in such a way that the incident field vanishes outside the grating coupler region. Namely, 


$$
\begin{aligned}
H_{2}^{(i)}\left(x_{1}, x_{3}\right)= & \exp \left[i \frac{\omega}{c}\left[\left(x_{1}-x_{0}\right) \sin \theta_{0}-x_{3} \cos \theta_{0}\right]\right. \\
& \left.\times\left[1+w\left(x_{1}-x_{0}, x_{3}\right)\right]\right] \\
& \times \exp \left[-\frac{1}{W^{2}}\left[\left(x_{1}-x_{0}\right) \cos \theta_{0}+x_{3} \sin \theta_{0}\right]^{2}\right],
\end{aligned}
$$

where

$$
w\left(x_{1}, x_{3}\right)=\frac{c^{2}}{\omega^{2} W^{2}}\left[\frac{2}{W^{2}}\left(x_{1} \cos \theta_{0}+x_{3} \sin \theta_{0}\right)^{2}-1\right] .
$$

The period $d_{c}$ of the grating coupler is

$$
\frac{d_{c}}{\lambda}=\left(\frac{c \operatorname{Re}\left[k_{\mathrm{SP}}(\omega)\right]}{\omega}-\sin \theta_{0}\right)^{-1},
$$

where Re denotes the real part. Hence the first diffracted order coincides with a forward propagating SPP, whose wave vector is given within the impedance boundary condition approximation by

$$
k_{\mathrm{SP}}(\omega)=\frac{\omega}{c}\left(1-\frac{1}{\varepsilon(\omega)}\right)^{1 / 2} .
$$

Moreover, for $\theta_{0} \leqslant 0$ only the zeroth order lies in the radiative region of the spectrum. In this way, the efficiency of the photon-SPP coupling should be enhanced.

Then the SPP thus excited propagates along the flatimpedance section, impinging on the grating scatterer. As a result, the SPP is reflected, transmitted, and also scattered into propagating waves in the vacuum.

\section{Energy balance}

The incident beam is normalized so that its total, timeaveraged power flow is $P_{0}=c^{2} L_{2} /(8 \pi \omega)$, where $L_{2}$ is the length in the $x_{2}$ direction. Then the angular distribution of the scattered intensity, namely, the differential reflection coefficient, is given by

$$
\frac{\partial R_{p}}{\partial \theta_{s}}=\frac{1}{2 \pi} \alpha_{0}^{2}\left(q=\omega \sin \theta_{s} / c, \omega\right)\left|R_{p}\left(q=\omega \sin \theta_{s} / c, \omega\right)\right|^{2},
$$

where $\theta_{s}$ is the scattering angle measured clockwise with respect to the $x_{3}$ axis. $R_{p}(q, \omega)$ is obtained from Eq. (8). The total radiated energy $S$ is therefore

$$
S=\int_{-\pi / 2}^{\pi / 2} d \theta_{S} \frac{\partial R_{p}}{\partial \theta_{S}}
$$

Nevertheless, it is also necessary to account for the energy carried by the SPP. The total, time-averaged flux transported by a SPP described by

$$
H_{2}^{>}\left(x_{1}, x_{3} \mid \omega\right)_{\mathrm{SP}}=A \exp \left[i k_{\mathrm{SP}}(\omega) x_{1}-\beta_{0}(\omega) x_{3}\right],
$$

is, neglecting the contribution from inside the metal,

$$
P_{\mathrm{SP}}=\frac{c^{2} L_{2}}{8 \pi \omega} \frac{|A|^{2}}{2} \frac{k_{\mathrm{SP}}(\omega)}{\beta_{0}(\omega)},
$$

with

$$
\beta_{0}(\omega)=\frac{\omega}{c}[-\varepsilon(\omega)]^{-1 / 2} .
$$

Consequently, if the surface field on the leftmost and rightmost flat ends of the interface are of the form of Eq. (15), the reflection and transmission coefficients become, respectively,

$$
\begin{gathered}
R=\frac{\left|A_{r}\right|^{2}}{2} \frac{k_{\mathrm{SP}}(\omega)}{\beta_{0}(\omega)} . \\
T=\frac{\left|A_{t}\right|^{2}}{2} \frac{k_{\mathrm{SP}}(\omega)}{\beta_{0}(\omega)} .
\end{gathered}
$$

In Eqs. (17), $A_{r}$ and $A_{t}$ are the corresponding magnetic field amplitudes. The unitarity condition (energy conservation) thus reads

$$
S+R+T=1 \text {. }
$$

\section{Numerical calculations}

As mentioned above, the integral equation (5) is solved numerically by means of a quadrature scheme. For that purpose, the finite surface is discretized in accordance with the following criterion for the sampling interval $\Delta x_{1}$ : $\Delta x_{1}=\lambda / 20$ in the regions of flat surface impedance, $\Delta x_{1}=\min \left(\lambda / 20, d_{c} / 20\right)$ within the grating coupler, and $\Delta x_{1}=\min \left(\lambda / 20, d_{s} / 20\right)$ within the grating scatterer. The number of sampling points per region is $N_{\alpha}=L_{\alpha} / \Delta x_{\alpha}$ (the subscript $\alpha$ stands for $\alpha=f, c$, and $s$, respectively). The total number of points is $N=3 N_{f}+N_{c}+N_{s}$. Therefore, our numerically solving Eq. (5) translates into solving a $N \times N$ system of linear equations. This allows us to obtain the magnetic field evaluated at a set of points on the surface. Then the scattering amplitude $R_{p}(q, \omega)$ can be also computed through a discretized version of Eq. (8), which leads to the differential reflection coefficient by the use of Eq. (13).

The numerical calculations have been carried out on an ALPHA AXP-2100. The total number of points employed varies as needed in the range $N=1000-10000$. As an example, the computation time for a surface consisting of $N=2000$ is $5 \mathrm{~min}$. Energy conservation [Eq. (18)] is systematically checked, being within $2 \%$ error in all cases studied here in the absence of dissipative losses. In order to make sure that our truncating the total surface length in the numerical calculations does not give rise to inconsistent results, we have increased $L_{f}$, keeping the other lengths fixed, finding that the differences in the results are negligible. In what follows, we present the results obtained by means of the numerical procedure described above for the study of both the excitation of SPP by a grating coupler illuminated by a Gaussian beam and the scattering of such SPP by another 
grating for frequencies in the vicinity of the lower band edge in the SPP dispersion relation.

\section{SURFACE PLASMON POLARITON COUPLING}

First, we will focus on the excitation of SPP by a Gaussian beam impinging on a grating coupler; thus we set $s_{s}=0$ in Eq. (9), so that there is no grating scatterer on the right-hand side of the grating coupler hindering the propagation of the SPP. The metal occupying the lower half-space is characterized by Drude's dielectric function $\varepsilon(\omega)=1-\omega_{p}^{2} / \omega^{2}$, where $\omega_{p}=2 \pi / \lambda_{p}$ is the plasma frequency. The plasma wavelength is $\lambda_{p}=157.1 \mathrm{~nm}$, which results from fitting $\varepsilon(\omega)$ as given above to the experimental values of the real part of the dielectric function of silver in the visible. The period of the sinusoidal grating coupler, unless otherwise stated, is hereafter given by Eq. (11). It depends on the wavelength $\lambda$ and the angle of incidence $\theta_{0}$ of the incident beam, and on the wave vector $k_{\mathrm{SP}}(\omega)$ of the SPP supported by the vacuum-metal interface [Eq. (12)].

The procedure to determine the coupling and diffracted efficiencies, and the energy balance, is as follows. First, the results for the squared modulus of the transverse surface magnetic field $\left|H\left(x_{1}\right)\right|^{2}$ (see the solid curve in Fig. 5 below, and also Fig. 2 in Ref. 23) when a Gaussian beam is incident at $\theta_{0}$ on the grating coupler reveal that a SPP is excited, which propagates in the forward direction - to the right of the grating - along the flat surface-impedance region. In this regard, the existence of a strong resonance appearing in the squared modulus of the scattering amplitude [cf. Eq. (8)] at $q \approx k_{\mathrm{SP}}$ corroborates the existence of a forward propagating SPP. Since the metal is assumed to be lossless, the amplitude of this forward propagating SPP remains constant, except for a small oscillation due to the interference with the SPP reflected at the edge of the truncated surface. (The amplitude of this backward-propagating SPP is almost negligible, as deduced from the visibility of these oscillations and the fact that $R \approx 0$.) Hence the SPP coupling efficiency $\eta_{\mathrm{SP}}$ can be defined as $\eta_{\mathrm{SP}}=T$ [cf. Eqs. (17)]. Second, the angular distribution of scattered intensity, calculated from Eqs. (8) and (13), shows a strong peak at $\theta_{s}=\theta_{0}$ that corresponds to the zeroth diffraction order, its width being inversely proportional to the spatial width of the incident beam. This contribution yields the zeroth-order diffraction efficiency $\eta_{0}$. In addition, a small contribution arises near the grazing direction $\theta_{s} \lesssim 90^{\circ}$ due to SPP diffraction effects. However, when checking energy conservation, one should bear in mind that the small SPP edge-diffraction contribution $S_{\mathrm{SP}}$ comes from the energy transported by the SPP along the interface, which has already been accounted for by the coefficient $T$. Thus $S_{\mathrm{SP}}$ has to be subtracted from $S$. Therefore energy conservation is redefined: $R+T+S^{\prime}=\eta_{0}+\eta_{\mathrm{SP}}\left(S^{\prime}=S-S_{\mathrm{SP}}\right)$.

Recall that the metal has been considered lossless with the aim of providing accurate and reliable results in the sense of the energy conservation criterion. In a more realistic situation, dissipative losses in the metal should be taken into account. This would facilitate the elimination of spurious edgediffraction contributions by placing on both sides of the finite grating coupler flat segments slightly longer than the SPP mean free path. We will discuss this point below.

Let us first study the influence of $\theta_{0}$ on the coupling ef-

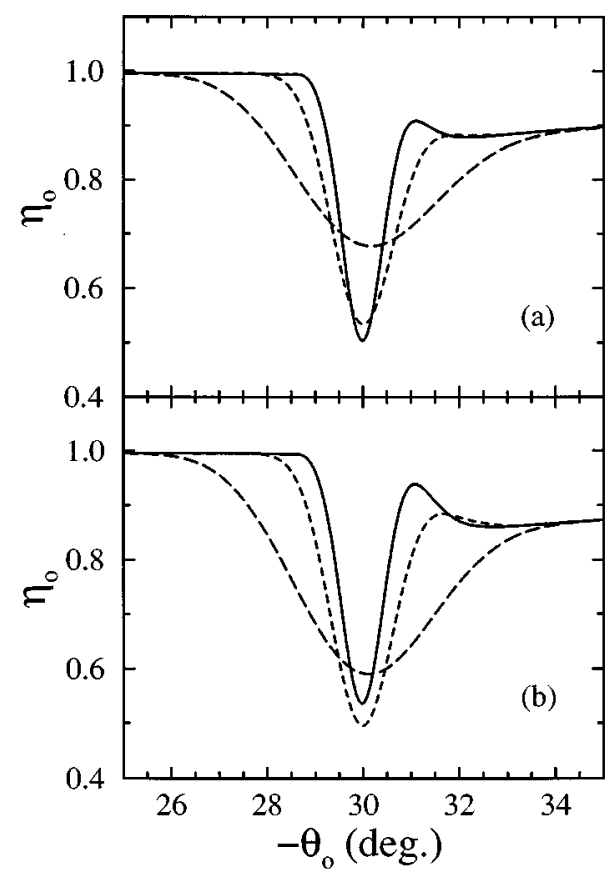

FIG. 2. Zeroth-order diffracted efficiencies as a function of the angle of incidence $\theta_{0}$ for a grating coupler with $s_{c}=1.2$. Solid curve, $N_{c}=150$; dashed curve, $N_{c}=100 ;$ long-dashed curve, $N_{c}=45$. (a) $\lambda=981.2 \mathrm{~nm}(\varepsilon=-38)$ and $d_{c}=648.6 \mathrm{~nm}$; (b) $\lambda=853.3 \mathrm{~nm}(\varepsilon=-28.5)$ and $d_{c}=562.4 \mathrm{~nm}$.

ficiency, denoted by $\eta_{\mathrm{SP}}=T$. In Fig. 2, the zeroth-order efficiencies versus $\theta_{0}$ are shown for $s_{c}=1.2$ and: (a) $\lambda=981.2 \mathrm{~nm}$ and (b) $\lambda=853.3 \mathrm{~nm}$. The coupler periods are, respectively, $d_{c}=648.6$ and $562.4 \mathrm{~nm}$, which are obtained from Eq. (11) with $\theta_{0}=-30^{\circ}$. For each value of the wavelength, three cases have been considered: $N_{c}=45,100$, and 150. The curves shown contain information about the SPP dispersion relation on the surface impedance under consideration. On the one hand, the position of the minimum (maximum) of the zeroth-order (coupling) efficiency accounts for the real part of the parallel component of the SPP wave vector $k_{\mathrm{SP}}(\omega)$ : Since there is no observable shift of these positions from $\theta_{0}=-30^{\circ}$ in Fig. 2 , the $\operatorname{Re}\left[k_{\mathrm{SP}}(\omega)\right]$ can be regarded for the set of frequencies and parameters used in obtaining the results shown in Fig. 2 as accurately given by the real part of the flat surface-impedance value [cf. Eq. (12)]. On the other hand, the coupling resonance width in $q$ space is proportional to the imaginary part of the SPP wave vector. Hence the wider the resonance, the smaller the SPP mean free path: in the absence of losses, the only contribution to $\operatorname{Im}\left[k_{\mathrm{SP}}(\omega)\right]$ (Im being the imaginary part) comes from the SPP-SPP and SPP-photon decay rates. Nonetheless, this resonance width is also limited by the incident-beam width in Fourier space, which constitutes in turn the prevailing mechanism for such narrow Gaussian beams as used in obtaining the results shown in Fig. 2. As expected from the fact that the beam width increases with $N_{c}$, the larger $N_{c}$ is the narrower is the coupling resonance width. Note the horizon for the first diffracted order at $\theta_{0}=-30.8^{\circ}$ and $\theta_{0}=-31.1^{\circ}$ in Figs. 2(a) and 2(b), respectively; these Rayleigh anomalies appear also more abruptly the larger $N_{c}$ is. 


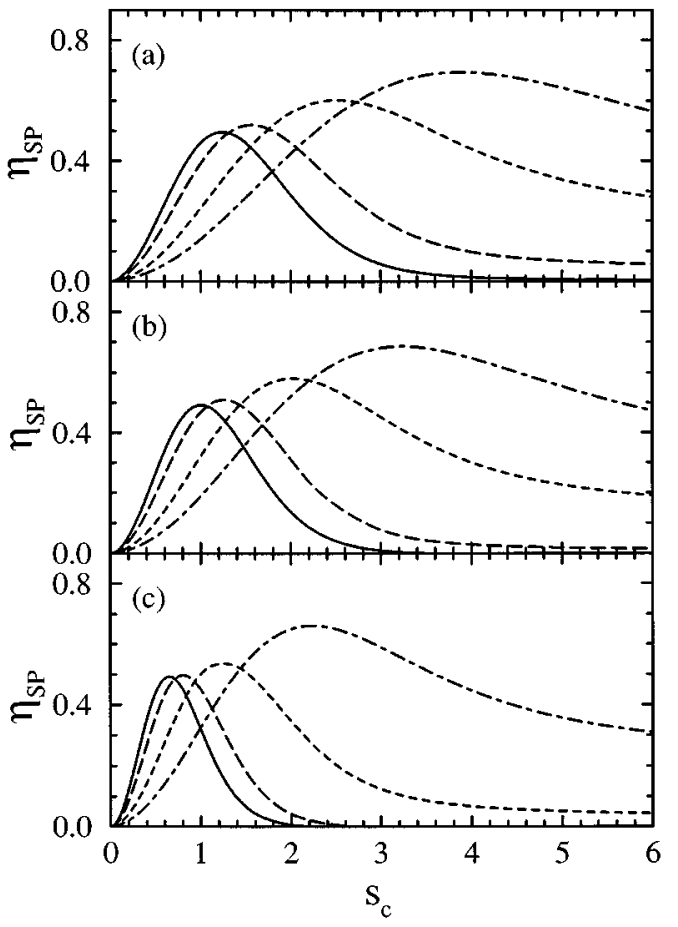

FIG. 3. Coupling efficiencies as a function of surface-impedance grating height $s_{c}$. Solid curve, $N_{c}=150 ;$ long-dashed curve, $N_{c}=100$; dashed curve, $N_{c}=45$; dot-dashed curve, $N_{c}=20$. (a) $\lambda=981.2 \mathrm{~nm}(\varepsilon=-38)$ and $d_{c}=648.6 \mathrm{~nm}$; (b) $\lambda=853.3 \mathrm{~nm}$ $(\varepsilon=-28.5)$ and $d_{c}=562.4 \mathrm{~nm}$; (c) $\lambda=633 \mathrm{~nm}(\varepsilon=-16.4)$ and $d_{c}=413.1 \mathrm{~nm}$.

We assume now that the grating height $s_{c}$ is varied whereas all of the other parameters remain fixed. The results thus obtained for the coupling efficiencies $\left(\eta_{\mathrm{SP}}\right)$ are shown in Fig. 3 for three values of the wavelength: (a) $\lambda=981.2$ $\mathrm{nm}$; (b) $\lambda=853.3 \mathrm{~nm}$; and (c) $\lambda=633 \mathrm{~nm}$. Four distinct values of the number of periods $N_{c}$ have been considered in every case: $N_{c}=20,45,100$, and 150 . The common features to all the coupling efficiencies are: rapid increase (slow decrease) for low (high) values of $s_{c}$, exhibiting a maximum at an intermediate value $s_{c}^{0}$. This behavior can be explained by means of the following argument. ${ }^{8}$ For very smooth surfaces, the grating allows the coupling of the incoming beam into a SPP, which then propagates with a long mean free path (note that in this case the metal is lossless); the larger the corrugation, the better the excitation is. When the grating height becomes sufficiently large, the probability of a SPP coupling back into light starts to grow, thus reducing the efficiency of the SPP excitation. Therefore, the maximum coupling observed in the curves of Fig. 3 appears as a compromise between both processes. Beyond that optimum height of maximum coupling, the SPP radiative damping gradually predominates over the excitation, leading to a decrease of the coupling efficiency. It is also of interest to analyze the influence of the number $N_{c}$ of periods in Fig. 3. As $N_{c}$ increases, the position of the maximum coupling efficiency shifts towards lower values of the grating height $s_{c}$. This reveals that shortening the grating coupler (equivalently, the illuminated spot size) reduces the radiative damping rate relatively faster than the excitation rate.

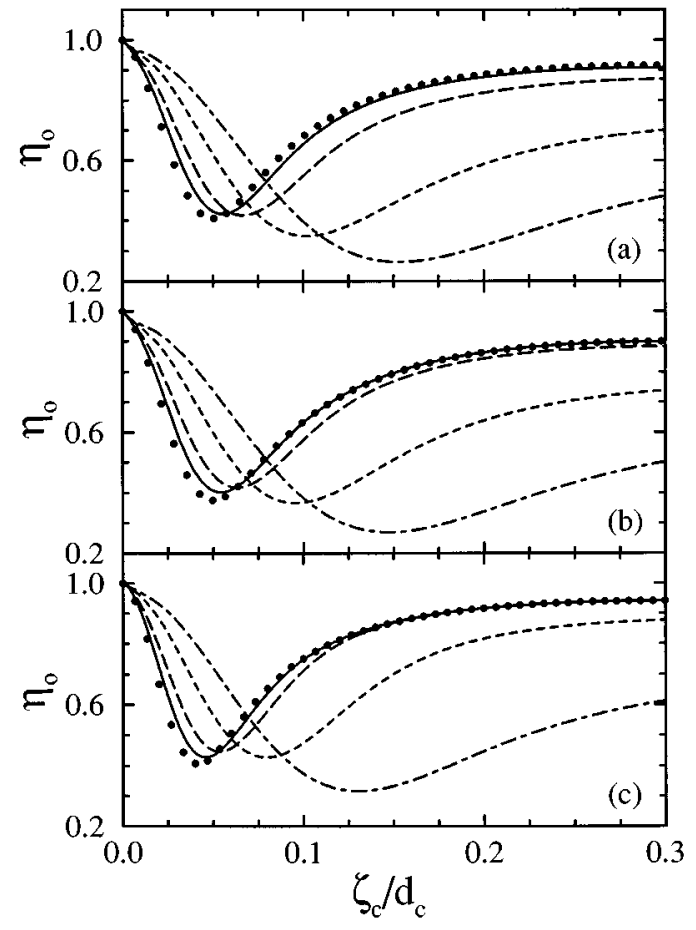

FIG. 4. Zeroth-order diffracted efficiencies as a function of the ratio $\zeta_{c} / d_{c}$ (real corrugation grating height over period). Dots, $N_{c}=200 ;$ solid curve, $N_{c}=150$; long-dashed curve, $N_{c}=100$; dashed curve, $N_{c}=45$; dot-dashed curve, $N_{c}=20$. (a) $\lambda=981.2 \mathrm{~nm}$ $(\varepsilon=-38+i 2.5) \quad$ and $\quad d_{c}=648.6 \quad \mathrm{~nm} ; \quad$ (b) $\lambda=853.3 \mathrm{~nm}$ $(\varepsilon=-28.5+i 1.94)$ and $d_{c}=562.4 \quad \mathrm{~nm} ; \quad$ (c) $\lambda=633 \mathrm{~nm}$ $(\varepsilon=-16.4+i 0.5)$ and $d_{c}=413.1 \mathrm{~nm}$.

On the other hand, note that the maximum value of the coupling efficiency saturates beyond $N_{c} \gtrsim 100$ at $\eta_{\mathrm{SP}} \approx 50 \%\left(\eta_{0} \approx 50 \%\right)$. In contrast, it is well known that zeroth-order efficiencies close to zero can be theoretically and experimentally obtained. ${ }^{8-12}$ Once again, one has to bear in mind that the results shown in Fig. 3 correspond to a lossless metal. In this connection, we have calculated $\eta_{0}$ versus $s_{c}$ as in Fig. 3 but taking into account the imaginary part of the dielectric function $\varepsilon_{I}$ of silver at the corresponding frequencies (cf. Ref. 38). The results reveal that the positions $s_{c}^{0}$ at which $\eta_{0}$ is minimum do not differ appreciably from those shown in Fig. 3. However these minimum values $\eta_{0}^{\min }$ are smaller when dissipative losses are included provided that $N_{c} \geqslant 100$, and tend to become even smaller as the length of the illuminated grating is further increased. In Fig. 4 , some of these results for the zeroth-order efficiencies are shown as a function of the renormalized scale $\zeta_{c} / d$, where we have used the relationship [cf. Eq. (3)] between surface impedance $s\left(x_{1}\right)$ and surface roughness $\zeta\left(x_{1}\right)$ established by Maradudin. ${ }^{34}$ In this manner, it is seen that the almost invariant behavior of the diffracted efficiencies for different frequencies is maintained not only in the infinite grating limit, ${ }^{12}$ but also for finite gratings under conditions of small illuminated spot size.

The results for $\lambda=633 \mathrm{~nm}$ in Fig. 4(c) can be compared with the theoretical and experimental results of Ref. 12. The maximum coupling efficiency for $N_{c}=200$ occurring at $\zeta_{c}^{0} / d_{c} \approx 0.04$ corresponds to a surface height of $\zeta_{c}^{0} \approx 16 \mathrm{~nm}$, in 


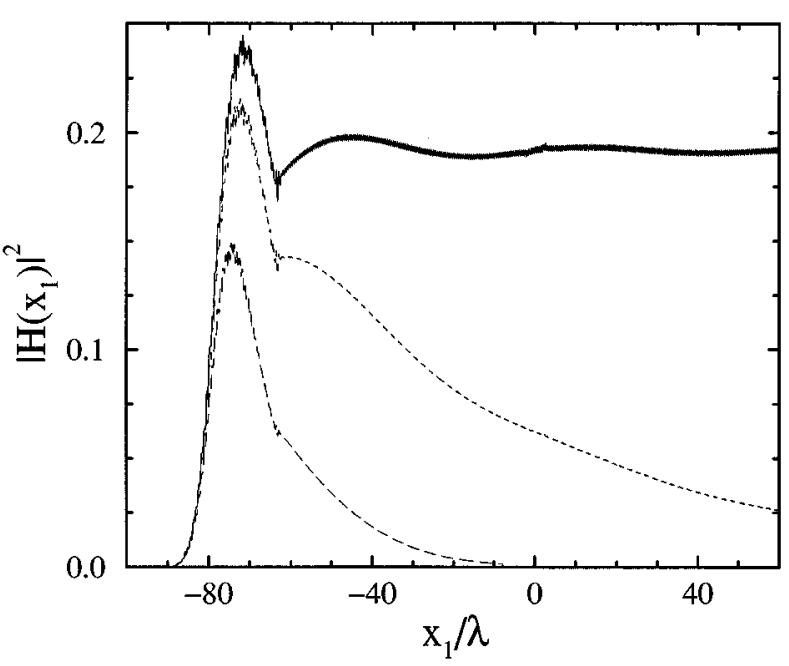

FIG. 5. Square modulus of the total surface magnetic field in arbitrary units resulting from illuminating $N_{c}=45$ periods of a grating coupler of period $d_{c}=562.4 \mathrm{~nm}$ and height $s_{c}=2$. The other parameters are $\lambda=853.3 \mathrm{~nm}, L_{f}=60 \lambda$, and $L_{s}=4.6 \lambda \quad\left(s_{s}=0\right)$. Solid curve, $\varepsilon=-28.5$; dashed curve, $\varepsilon=-28.5+i 1.94$; longdashed curve, $\varepsilon=-28.5+i 10$. The curves have been smoothed by averaging point by point over the period $d_{c}$.

agreement with the results shown in Fig. 5 of Ref. 12. This gives support to the use of a surface impedance boundary condition to simplify the scattering integral equations (cf. Sec. II), not only from a qualitative standpoint, but also as regards obtaining accurate quantitative results, inasmuch as the conditions for its validity are satisfied ${ }^{34}$ (note that these conditions are ensured for $\lambda=633 \mathrm{~nm}, d_{c}=413.1 \mathrm{~nm}$, and $\zeta_{c}^{0}=16 \mathrm{~nm}$ ). The disagreement of the calculated values of the zeroth-order efficiencies shown in Fig. 4(c) with respect to the experimental values given in Ref. 12 stem from the different number of periods illuminated by the incoming beam: $N_{c} \leqslant 200$ in our case versus $N_{c}=5000$ therein, in practice equivalent to an infinite grating.

Still the question remains open as to whether or not a lower zeroth-order efficiency leads to a higher SPP coupling efficiency when energy conservation is not guaranteed. To answer this question, we show in Fig. $5\left|H\left(x_{1}\right)\right|^{2}$ in three cases that differ from each other only in the value of $\varepsilon_{I}$ : (a) lossless metal $\varepsilon_{I}=0$; (b) real silver $\varepsilon_{I}=0.5$; and (c) very lossy metal $\varepsilon_{I}=10$. In all three cases, the other parameters are: $\lambda=633 \mathrm{~nm}, d_{c}=413.1 \mathrm{~nm}, s_{c}=2$, and $N_{c}=45$. The rapid oscillations appearing within the grating coupler region have been smoothed in Fig. 5 by averaging point by point over the period $d_{c}$. Apart from the fact that the amplitude of the excited SPP decays exponentially when $\varepsilon_{I} \neq 0$ on propagating along the flat surface, one can extract information about the output power carried by the SPP from the value of $\left|H\left(x_{1}\right)\right|^{2}$ at the right border of the grating coupler. From these values, it is evident that the SPP output power is higher the less lossy the metal is, despite the fact that $\eta_{0}$ decreases with $\varepsilon_{I}$. In other words, Figs. 3, 4, and 5 demonstrate that lower zeroth-order efficiencies do not necessarily indicate higher SPP coupling efficiencies when comparing different materials and/or frequencies, since dissipative effects become very important when resonance takes place. Note also the strong magnetic fields resonantly generated within the grating coupler, which are indeed larger than shown in Fig. 5 due to the fact that the oscillations of period $d_{c}$ have been smoothed. Enhancement factors of the order of 100 have been found in agreement with previous works; ${ }^{8-11}$ they diminish as dissipative losses increase, as expected. These factors play a crucial role in the surface-enhanced Raman spectroscopy of molecules adsorbed on such metallic gratings. ${ }^{13}$

\section{SURFACE PLASMON POLARITON SCATTERING}

Let us now study the scattering of a SPP by another sinusoidal grating placed in its propagation path on the metal surface [cf. Eq. (9)]. The scatterer grating period and height throughout this section will be $d_{s}=392.5 \mathrm{~nm}$ and $s_{s}=3$, respectively. As a result of the scattering, the incoming SPP is partially reflected, transmitted, and converted into radiating modes into the vacuum half-space. This process is fundamentally ruled by the frequency dependence of the SPP wave vector $k_{\mathrm{SP}}(\omega)$ on the grating parameters $d_{s}$ and $s_{s}$. Thus, before going on to the finite grating case we are interested in, it is necessary to calculate the SPP dispersion relation for an infinite sinusoidal grating impedance described by the parameters $d_{s}$ and $s_{s}$ given above. This has been done numerically by looking for the zeros of the determinant of the matrix resulting from imposing the impedance boundary condition (2) on the magnetic field amplitude written in the form of a truncated Rayleigh expansion. ${ }^{27}$ The results thus obtained, not shown here, reveal that a gap in the propagation of SPP opens up for frequencies $\omega$ such that $0.45<\omega /(c G)<0.4945\left(G=2 \pi / d_{s}\right)$, separating the first two allowed bands. In what follows, we focus on two frequencies: $\quad \omega /(c G)=0.44 \quad(\lambda=892.0 \quad \mathrm{~nm}), \quad$ and $\omega /(c G)=0.46(\lambda=853.3 \mathrm{~nm})$, which lie, respectively, in the band and in the gap of the SPP dispersion relation for the infinite grating. Note that both frequencies are close to the lower band edge, where the interesting finite-scale effects are more clearly manifested.

In order to excite a SPP of the required frequency, a grating coupler of period $d_{c}$ defined by Eq. (11) and consisting of $N_{c}=45$ periods is illuminated by a Gaussian beam as described in the preceding section. The energy conservation criterion for the entire process-coupling + scattering-is satisfied in all the cases shown below within $2 \%$ error, as mentioned in Sec. II. Specifically, since we are now interested in the scattering of the SPP by the second grating, we proceed as follows: First, the SPP transmission coefficient $T_{0}$ is calculated in the absence of grating scatterer $\left(s_{s}=0\right)$, keeping all the other parameters fixed. $T_{0}$ yields the squared modulus of the amplitude of the incoming SPP. Then the normalized SPP reflection and transmission coefficients associated with the interaction of the SPP with the second grating are obtained by dividing $R$ and $T$, respectively, resulting from the entire process with grating scatterer $\left(s_{s} \neq 0\right)$, by $T_{0}$. Finally, the total radiated intensity due to the conversion of the SPP into volume waves at the grating scatterer can be deduced from energy conservation arguments. It should be emphasized, however, that in some cases one may be interested in the entire process as, for instance, when a filter is placed sufficiently close to a coupler. Under such circumstances, our numerical results based on the scattering integral 


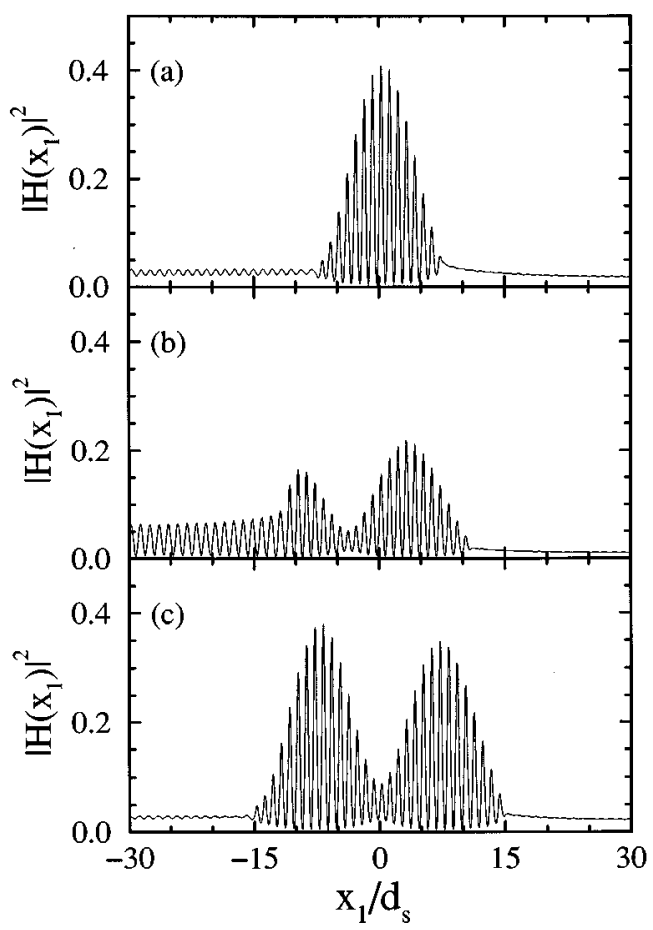

FIG. 6. $\left|H\left(x_{1}\right)\right|^{2}$ versus $x_{1} / d_{s}$ for $\lambda=892 \mathrm{~nm}, d_{c}=588.5 \mathrm{~nm}$, $s_{c}=0.5, N_{c}=45, \varepsilon=-31.2, \theta_{0}=-30^{\circ}$, and $L_{f}=10 \lambda$, with the presence of a grating scatterer of period $d_{s}=392.5 \mathrm{~nm}$ [so that $\omega /(c G)=0.44]$ and height $s_{s}=3$. (a) $N_{s}=15$, (b) $N_{s}=22$, (c) $N_{s}=30$.

equation formulation yield straightforwardly the relevant physical magnitudes of the problem.

\section{A. Frequency in the band: Resonance transmission}

Figure 6 shows $\left|H\left(x_{1}\right)\right|^{2}$ for $s_{c}=0.5$ in the region within the grating scatterer, and its vicinity, for three values of the number of periods: (a) $N_{s}=15$; (b) $N_{s}=22$; and (c) $N_{s}=30$. As is seen in Fig. 6, strong oscillations producing significant field enhancements appear within the grating scatterer, symmetrically located about $x_{1}=0$, that follow the sinusoidal profile of the surface impedance. In this regard, the field enhancements obtained within the grating have been found to be larger the closer the frequency is to the band edge. As a result of the scattering process, the incoming SPP is partially transmitted (see Fig. 6 for $x_{1} \geqslant N_{s} d_{s} / 2$ ) and reflected (see Fig. 6 for $x_{1} \leqslant-N_{s} d_{s} / 2$ ). The reflected SPP can be traced along the surface through its interference with the incoming SPP within the flat section between the coupler and the scatterer. Moreover, although not shown in Fig. 6, the reflected SPP interferes with the magnetic field on the grating coupler producing a complicated pattern, appearing as the outcoming SPP on the left-hand side of the coupler. As mentioned above, the constant field squared moduli at the leftmost and rightmost flat sections of the entire surface yield the SPP reflection and transmission coefficients, respectively.

In Fig. 7, the angular distribution of radiated intensity is shown for the same case as in Fig. 6(b). Apart from the contributions (zeroth order and edge diffraction) described in the preceding section, the effects of the scattering process are

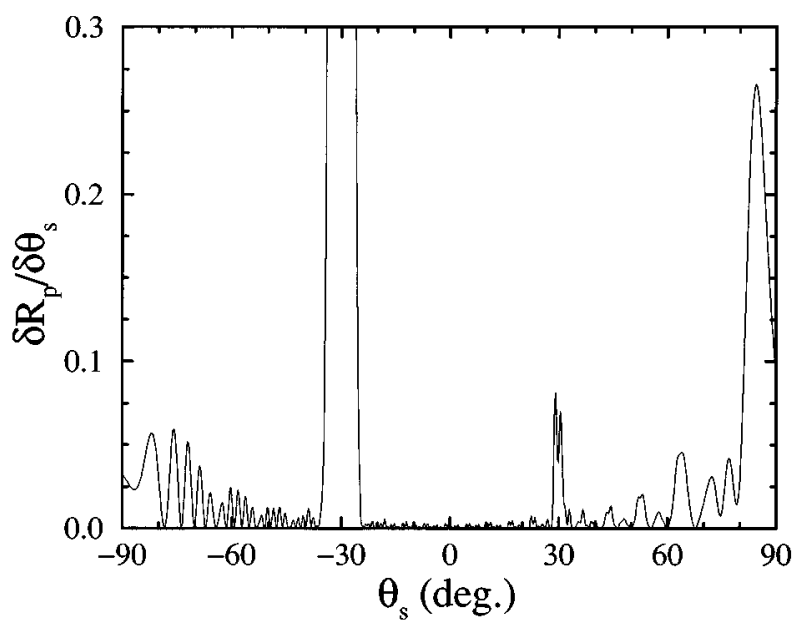

FIG. 7. Angular distribution of scattered intensity for the same scattering geometry as in Fig. 6(b).

evident in the contributions arising near grazing scattering angles $\left|\theta_{s}\right| \leqslant 90^{\circ}$. In addition, the tiny peak at $\theta_{s}=30^{\circ}$ reveals that a small fraction of the reflected SPP couples back into propagating waves through its interaction with the grating coupler. Note that, in the absence of reflected SPP, neither the peak at $\theta_{s}=30^{\circ}$ nor the contribution at negative grazing scattering angles ought to be present, as our results have confirmed (although not shown here).

Let us now return to the analysis of the reflection and transmission coefficients as derived from $\left|H\left(x_{1}\right)\right|^{2}$ in Fig. 6. Note that the magnetic field inside the grating scatterer $\left(\left|x_{1}\right| \leqslant N_{s} d_{s} / 2\right)$ presents a slowly varying envelope modulating the high-frequency oscillations mentioned above. The spatial frequency of this envelope-divided by a factor of 2 owing to the squared modulus-yields the crystal momentum of the SPP mode on an infinite grating. Since the frequency of the incoming SPP matches that of the SPP mode $k_{\mathrm{SP}}^{\text {cryst }}$ close below the lower band edge of the SPP band structure, the real part of the crystal momemtum is consequently small. (In fact, it tends to zero as the frequency approaches the band gap.) Therefore the surface field structures in Figs. $6(\mathrm{a}), 6(\mathrm{~b})$, and $6(\mathrm{c})$ demonstrate that the grating lengths of $L_{s} / d_{s}=15,22$, and 30 periods, respectively, approximately correspond to $1 / 2,3 / 4$, and 1 period of the SPP crystal mode. If we now represent the normalized transmission and reflection coefficients as a function of the number of grating periods (see Fig. 8), similar oscillations are observed. It turns out that maximum resonant SPP transmission is achieved when the length of the grating matches an integer number of half the period of the SPP crystal mode $\left(L_{s}=n \pi / k_{\mathrm{SP}}^{\text {cryst }}\right.$, $n=1,2,3, \ldots)$. On the other hand, the incident SPP is more strongly reflected-minimum transmission-when the grating length lies between those values of resonant transmission lengths. The radiated energy might represent a substantial part of the energy $(\sim 30 \%)$, but it originates to a large extent in both edge-diffraction effects and grating-induced SPPphoton coupling. The independence of the normalized transmission coefficient from the intensity of the incoming SPP is demonstrated by considering another height $s_{c}$ of the illuminated grating coupler: the result obtained for $s_{c}=2$, not 


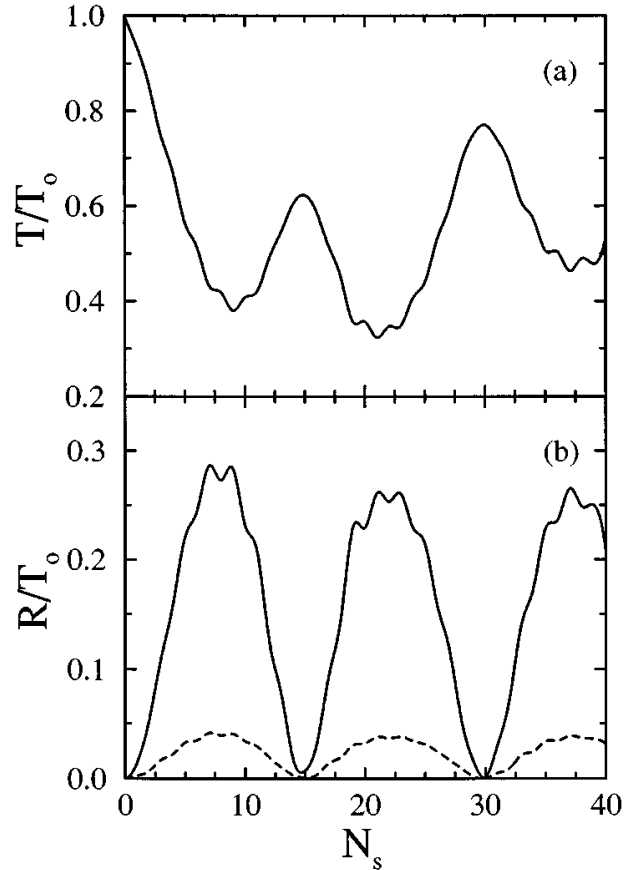

FIG. 8. Normalized (a) transmission and (b) reflection coefficients as a function of the number of grating scatterer periods $N_{s}$ $\left(d_{s}=392.5 \mathrm{~nm}\right.$ and $s_{s}=3$ ). (a) $s_{c}=0.5$. (b) Solid curve, $s_{c}=0.5$; dashed curve, $s_{c}=2$. The other parameters are $\lambda=892 \mathrm{~nm}$ $[\omega /(c G)=0.44], d_{c}=588.5 \mathrm{~nm}, N_{c}=45, \varepsilon=-31.2, \theta_{0}=-30^{\circ}$, and $L_{f}=10 \lambda$.

shown here, coincides with that of Fig. 8(a) for $s_{c}=0.5$. However, since $R / T_{0}$ underestimates the true reflection coefficient-part of the reflected SPP couples back into a diffracted peak at $\theta_{s}=30^{\circ}$ on passing through the couplerthere exist differences in the normalized reflection coefficient [see Fig. 8(b)]; the SPP-photon coupling for $s_{c}=2$ is relatively larger than for $s_{c}=0.5$, thus producing a smaller $R / T_{0}$ in the case of $s_{c}=2$. To emphasize the existence of strong finite-scale effects, we would like to stress the fact that significant reflection coefficients, of the order of $30 \%$ (actually, an estimate of $60 \%$ or larger is made if the reflected SPP reradiation contributions are considered), are found in spite of the frequency lying within the band of the SPP dispersion relation, for which one would expect total transmission. This is not surprising, however, if it is recalled that the finite grating scatterer behaves as a Fabry-Perot interferometer, or, similarly, as a resonance scattering potential barrier. ${ }^{39}$ It should be mentioned that other finite-scale effects in the frequency dependence of the transmission coefficient have been shown elsewhere. ${ }^{28}$

\section{B. Frequency in the gap: Tunneling}

Figure 9 shows $\left|H\left(x_{1}\right)\right|^{2}$ for $s_{c}=0.5$ and $N_{s}=4,8$, and 12 periods, within the grating scatterer $\left(\left|x_{1}\right| \leqslant N_{s} d_{s} / 2\right)$, Leaving aside the fine structure related to the rapid oscillations commented on above [see Fig. 6], we observe in the envelope of the squared modulus of the magnetic field strong enhancements about the first two periods, followed by a rapid decay. This decay is approximately exponential, as expected, and its decay length must be inversely proportional to the imaginary

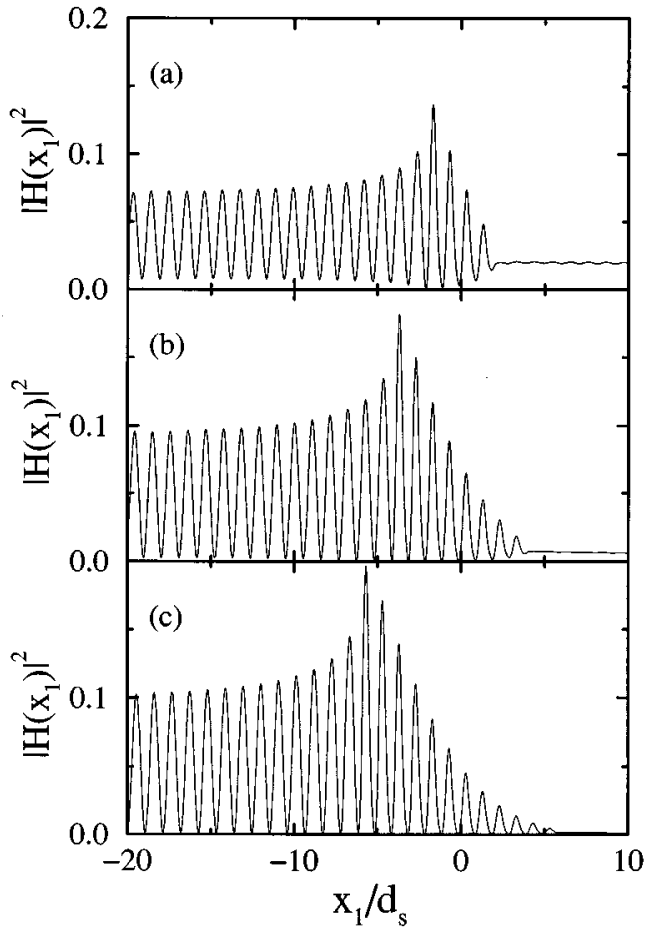

FIG. 9. Same as in Fig. 6 but for $\lambda=853.3 \mathrm{~nm}$ $[\omega /(c G)=0.46], \varepsilon=-28.5$, and $d_{c}=562.4 \mathrm{~nm}$. (a) $N_{s}=4$, (b) $N_{s}=8$, (c) $N_{s}=12$.

part of the SPP crystal momemtum (its real part should vanish). Despite the fact that the frequency lies in the gap, when the length of the grating is sufficiently small the SPP can tunnel through the structure as revealed in Figs. 9(a) and 9(b), leading to a significant amount of transmitted SPP. The dependence of the SPP tunneling intensity on the barrier length can be studied by plotting the normalized transmission coefficient as a function of the grating length in units of the period $\left(L_{s} / d_{s}=N_{s}\right)$. This is done in Fig. 10(a) for $s_{c}=0.5$. As in Fig. 8(a), the result obtained by means of a different grating coupler height $\left(s_{c}=2\right.$, not shown here) is indistinguishable from that for $s_{c}=0.5$. The reflection coefficient is also plotted for two values of $s_{c}$ [see Fig. 10(b)]. We observe, regardless of the input power, a rapid decay of $T$ as $N_{s}$ increases up to $N_{s}=12$, beyond which tunneling becomes negligible and the incoming SPP is reflected back $\left(R / T_{0} \approx 60 \%\right)$. The remaining energy $(\sim 40 \%)$ is scattered into propagating modes in the vacuum. The decay of $T$ slightly departs from a negative exponential, possibly due to both radiative losses and the transient regime occurring for very short grating lengths $\left(N_{s} \lesssim 4\right)$.

\section{CONCLUSIONS}

In summary, we have applied the exact Green's-theorem integral equation formalism for the scattering of electromagnetic waves from a corrugated surface to study both the coupling of a Gaussian beam into a surface plasmon polariton through a finite, sinusoidal metal grating coupler, and the subsequent scattering of the SPP thus excited by another sinusoidal grating of finite length. A planar-surface impedance boundary condition has been used to write the normal 


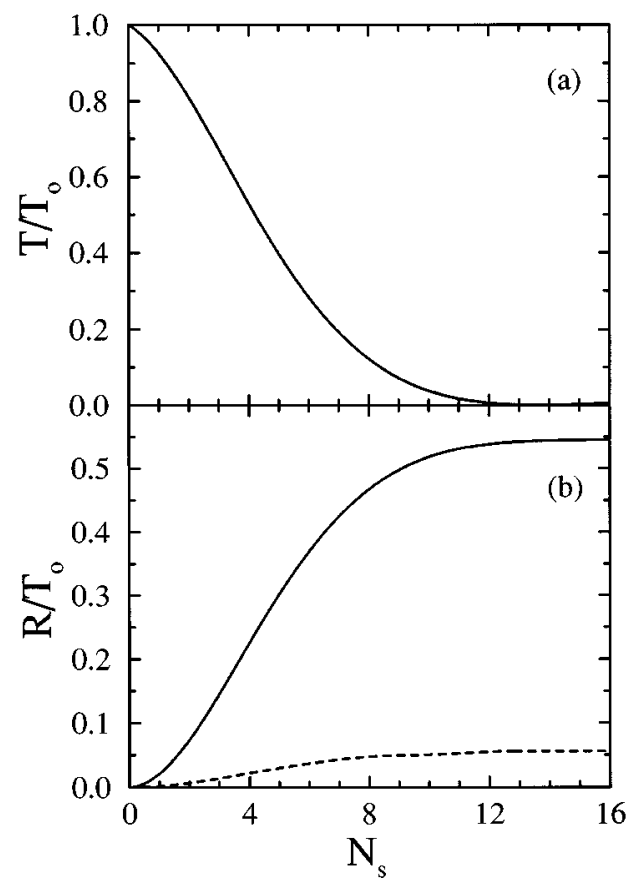

FIG. 10. Same as in Fig. 8 but for $\lambda=853.3 \mathrm{~nm}$ $[\omega /(c G)=0.46], \varepsilon=-28.5$, and $d_{c}=562.4 \mathrm{~nm}$.

derivative of the magnetic field on the surface in terms of the surface magnetic field itself. In this manner, the integral equations are simplified, leading to a considerable reduction of the computation time and memory required in the numerical procedure, while still providing reliable results for the range of parameters studied here. In particular, the magnetic field on the surface, the angular distribution of the scattered intensity, and the angular spectrum in the nonradiative region have been calculated. Energy balance is controlled not only by obtaining the entire energy radiated into the vacuum halfspace, but also by calculating the power flow carried by the SPP from its amplitude at the surface edges.

As regards the excitation of SPP through a grating for which only the zeroth order is a propagating one, finite-scale effects have been addressed in the dependence of the zeroth order and coupling efficiencies on the angle of incidence $\theta_{0}$ and the grating height $s_{c}$. Upon varying $\theta_{0}$, it has been shown that the resonance becomes narrower as the illuminated length of the grating coupler increases, provided that this length does not exceed the corresponding SPP damping length for the metal surface under consideration. No appreciable angular shifts are observed in our calculations for the frequencies and coupler parameters dealt with, indicating that the real part of the wave vector of the SPP propagating along the finite grating couplers studied here does not differ appreciably from its value for a planar surface. Our results for the coupling efficiency as a function of the grating height manifest a broadening of the resonance with decreasing illuminated coupler length; moreover, a shift of the position of the maximum coupling efficiency towards larger values of the surface height is observed when the coupler length decreases $\left(N_{c} \leqslant 150\right)$. In addition, the influence of dissipative losses inside the metal has been studied by arbitrarily modifying the imaginary part of the dielectric function, keeping the real part fixed. It has been shown that, despite the fact that the zeroth-order efficiency for a lossy metal, becomes smaller than that for a lossless (or less lossy) metal, apparently indicating a stronger coupling efficiency, the SPP power output escaping from the grating coupler is nevertheless larger the less lossy the metal is. The reason why this occurs is obviously the total conversion of the energy not contained in the zeroth diffracted order into SPP in a lossless metal (actually, a coupling efficiency of $\sim 50 \%$ is found that manifests the interplay between SPP excitation and reradiation mechanisms); in contrast, absorption is resonantly enhanced in a lossy metal, thus highly reducing the effective photon-SPP conversion rate. In this regard, large field enhancements $(\sim 100)$ appear when the photon-SPP coupling peaks, being larger the less lossy the metal is.

The reflection and transmission of SPP, and their conversion into volume waves, by a finite sinusoidal metallic grating has also been studied for two frequencies of the incident EM wave, which lie in the band and in the gap, respectively, of the SPP dispersion relation. These frequencies are close enough to the lower band edge so that finite-scale effects are encountered. When the frequency lies in the band, the reflection and transmission coefficients show a strongly oscillatory behavior as functions of the grating scatterer length in such a way that maximum transmission occurs when the number of periods coincides resonantly with an integer number of half the period of the SPP crystal mode. This resonant transmission is accompanied by moderate field enhancements $(\sim 4)$. When the frequency lies in the gap, it is found that the SPP can tunnel through the grating provided that its width is sufficiently small $\left(N_{s} \leqslant 10\right.$ in this case). The decay of the transmission coefficient has been calculated as a function of the barrier length. Beyond $N_{s}=12$ the grating reflects a large part ( $\sim 60 \%$ in the absence of dissipative losses) of the incoming SPP power flow, except for a significant amount of energy $(\sim 40 \%)$ that is radiated into the vacuum.

We would like to stress the point here that the emphasis in this work has been put on the study of diffraction by metal gratings with confined geometries, for which the numerical treatment based on the exact integral equation formulation gives rigorous results. These geometries might be of interest in practical applications such as couplers, filters, and other optical devices not only as concerns SPP, but also for other kind of surface waves (polaritons). On the other hand, it should be mentioned that the electromagnetic field at any point above the metal surface can be straightforwardly calculated from the surface field [cf. Eq. (4)]; this could provide valuable information in the experimental and theoretical investigation of the propagation and localization of SPP in near-field optics. ${ }^{29-32}$

\section{ACKNOWLEDGMENTS}

The author would like to thank Professor A. A. Maradudin for helpful discussions during the course of this work, and Professor M. Nieto-Vesperinas for critical reading of the manuscript. Financial support from the Spanish Consejo Superior de Investigaciones Científicas and the Dirección General de Investigación Científica y Técnica (Grant No. PB930973-C02) is acknowledged. This work was supported in part by Army Research Office Grant No. DAAL 03-92-G0239 . 
${ }^{1}$ R. W. Wood, Philos. Mag. 4, 396 (1902).

${ }^{2}$ Lord Rayleigh, Proc. R. Soc. London Ser. A 79, 399 (1907).

${ }^{3}$ U. Fano, J. Opt. Soc. Am. 31, 213 (1941).

${ }^{4}$ A. Hessel and A. A. Oliner, Appl. Opt. 4, 1275 (1965).

${ }^{5}$ Electromagnetic Theory of Gratings, edited by R. Petit (SpringerVerlag, Berlin, 1980).

${ }^{6}$ D. Maystre, in Progress in Optics, edited by E. Wolf (NorthHolland, Amsterdam, 1984), Vol XXI.

${ }^{7}$ D. Maystre and R. Petit, Opt. Commun. 17, 196 (1976); M. C. Hutley and D. Maystre, ibid. 19, 431 (1976).

${ }^{8}$ D. L. Mills and M. Weber, Phys. Rev. B 26, 1075 (1982); M. Weber and D. L. Mills, ibid. 27, 2698 (1982).

${ }^{9}$ M. Neviere and R. Reinisch, Phys. Rev. B 26, 5403 (1982).

${ }^{10}$ N. García, Opt. Commun. 45, 307 (1983).

${ }^{11}$ E. Popov and L. Tsonev, Opt. Commun. 69, 193 (1989); E. Popov, L. Tsonev, and D. Maystre, J. Mod. Opt. 37, 379 (1990).

${ }^{12}$ S. H. Zaidi, M. Yousaf, and S. R. J. Brueck, J. Opt. Soc. Am. B 8, 770 (1991).

${ }^{13}$ See, for example, Surface Enhanced Raman Scattering, edited by R. K. Chang and T. E. Furtak (Plenum, New York, 1981).

${ }^{14}$ D. Maystre, in Electromagnetic Theory of Gratings (Ref. 5), Chap. 3.

${ }^{15}$ See, for example, Progress in Optics (Ref. 6), pp. 49-52.

${ }^{16}$ J. M. Soto-Crespo and M. Nieto-Vesperinas, J. Opt. Soc. Am. A 6, 367 (1989).

${ }^{17}$ A. Sentenac and J.-J. Greffet, J. Opt. Soc. Am. A 9, 996 (1992).

${ }^{18}$ P.-P. Borsboom and H. J. Frankena, J. Opt. Soc. Am. A 12, 1134 (1995); 12, 1142 (1995).

${ }^{19}$ A. A. Maradudin, T. Michel, A. R. McGurn and E. R. Méndez, Ann. Phys. (N.Y.) 203, 255 (1990).

${ }^{20}$ M. Saillard and D. Maystre, J. Opt. Soc. Am. A 7, 982 (1990).

${ }^{21}$ J. A. Sánchez-Gil and M. Nieto-Vesperinas, J. Opt. Soc. Am. A 8, 1270 (1991); Phys. Rev. B 45, 8623 (1992).

${ }^{22}$ M. Nieto-Vesperinas, Scattering and Diffraction in Physical Optics (Wiley, New York, 1991).
${ }^{23}$ M. Saillard, Opt. Commun. 96, 1 (1993).

${ }^{24}$ Surface Polaritons on Smooth and Rough Surfaces and on Gratings, edited by H. Raether (Springer-Verlag, Berlin, 1988).

${ }^{25}$ Surface Polaritons, edited by V. M. Agranovitch and D. L. Mills (North-Holland, Amsterdam, 1982).

${ }^{26}$ F. Pincemin, A. Sentenac, and J.-J. Greffet, J. Opt. Soc. Am. A 11, 1117 (1994); F. Pincemin, A. A. Maradudin, A. D. Boardman, and J.-J. Greffet, Phys. Rev. B 50, 15261 (1994).

${ }^{27}$ A. A. Maradudin and J. A. Sánchez-Gil (unpublished).

${ }^{28}$ F. Pincemin and J.-J. Greffet, J. Opt. Soc. Am. A (to be published).

${ }^{29}$ U. Ch. Fisher and D. W. Pohl, Phys. Rev. Lett. 62, 458 (1989).

${ }^{30}$ P. M. Adam, L. Salomon, F. de Fornel, and J. P. Goudonnet, Phys. Rev. B 48, 2680 (1993); P. Dawson, F. de Fornel, and J. P. Goudonnet, Phys. Rev. Lett. 72, 2927 (1994).

${ }^{31}$ A. Madrazo and M. Nieto-Vesperinas, J. Opt. Soc. Am. A 12, 1298 (1995); ibid. (to be published).

${ }^{32}$ See, for example, Near Field Optics, edited by D. W. Pohl and D. Courjon (Kluwer, The Netherlands, 1993).

${ }^{33}$ P. J. Valle, F. Moreno, J. M. Sáiz, and F. González, Phys. Rev. B 51, 13681 (1995).

${ }^{34}$ A. A. Maradudin, in Topics in Condensed Matter Physics, edited by M. P. Das (Nova, New York, 1994).

${ }^{35}$ R. Depine, J. Mod. Opt. 34, 1135 (1987); J. Opt. Soc. Am. A 5, 507 (1988).

${ }^{36}$ R. García-Molina, A. A. Maradudin, and T. A. Leskova, Phys. Rep. 194, 351 (1990); A. A. Maradudin, Opt. Commun. 103, 227 (1993).

${ }^{37}$ A. A. Maradudin and E. R. Méndez, Physica A 207, 302 (1994).

${ }^{38}$ D. W. Lynch and W. R. Hunter, in Handbook of Optical Constants of Solids, edited by E. D. Palik (Academic Press, New York, 1985), p. 356.

${ }^{39}$ C. Cohen-Tannoudji, B. Diu, and F. Laloë, Quantum Mechanics (Wiley, New York, 1977), Vol. I, pp. 72-74. 